\title{
Radiation doses and risks to neonates undergoing radiographic examinations in intensive care units in Tunisia
}

\author{
Abir Bouaoun1,2, Latifa Ben-Omrane², Azza Hammou² \\ ${ }^{1}$ Université de Tunis El Manar, Institut Supérieur des Technologies Médicales, LR13ES07 laboratoire de Biophysique \\ et technologies médicales, Tunis, Tunisia \\ ${ }^{2}$ National Center of Radiation Protection, Tunis, Tunisia
}

Received May 01, 2015; Revised June 18, 2015; Accepted June 24, 2015; Published Online September 07, 2015

\section{Original Article}

\begin{abstract}
Purpose: To assess the radiation doses to neonates from diagnostic radiography in order to derive the local diagnostic reference levels (LDRLs) for optimisation purposes. Methods: This study was carried out in the neonatal intensive care units (NICU) of two hospitals in Tunis. 134 babies, with weights ranging from $635 \mathrm{~g}$ to $6680 \mathrm{~g}$, performed chest-abdomen X-ray examinations. Neonates were categorized into groups of birth weight. For each X-ray examination, patient data and exposure parameters were recorded. Dose area product (DAP) was measured and entrance surface dose (ESD) was estimated. Effective dose was calculated from the Monte Carlo simulation software PCXMC. Results: DAP values increased with neonatal weight and demonstrated a wide variation $\left(5.0-43.0 \mathrm{mGy} . \mathrm{cm}^{2}\right.$, mean $\left.23.4 \mathrm{mGy} . \mathrm{cm}^{2}\right)$ for patient weight from $600 \mathrm{~g}$ to $4000 \mathrm{~g}$. A wide variation was also observed for ESD (14 - $93 \mu \mathrm{Gy}$, mean $55.2 \mu \mathrm{Gy}$ ). The LDRLs expressed in term of DAP were estimated to be $17.6 \mathrm{mGy}^{\mathrm{c}} \mathrm{cm}^{2}$ and $29.1 \mathrm{mGy} . \mathrm{cm}^{2}$ for the first and the second NICU, respectively. In terms of effective dose, the average value was about $31.6 \mu \mathrm{Sv}$ single radiological examination. The results show the necessity to use a standardized protocol with high voltage technique combined to lower current time product ( $\mathrm{mAs}$ ) values and an adapted collimation which could lead to further reductions in the neonatal doses. Conclusion: This study presents the LDRLs and the effective doses for neonates in two NICUs and demonstrates the necessity to optimize patient protection for this category of patient.
\end{abstract}

Keywords: Patient Dose; Diagnostic Radiology; Neonatology; DAP; Effective Dose; LDRL

\section{Introduction}

Sick and premature neonates present a number of challenges in regards to their health. In the neonatal intensive care units (NICU), neonates, and especially those born prematurely often suffer from serious medical complications, due to diseases in the respiratory and cardiovascular system. During their hospitalization in these units, most of them will require multiple chest and chest abdomen radiologic examinations, for the diagnosis, the follow-up and the treatments. In fact, radiation risk relies mainly on the patient's age; therefore neonates are at highest risk for radiation induced malignancies ${ }^{1,2}$ as a consequence of relatively long life expectancy which together with their radiosensitivity and potentially large number of radiographs performed during a short period of time. It is approximately believed that the risk of cancer induction per unit of dose is 2-3 times higher than that of the adult population and 6-9 times higher than the risk from an exposure of a 60 -year-old. ${ }^{3}$ It is therefore important to ensure that radiation doses from radiographic examinations carried out in neonatal units are kept at a minimum whilst maintaining the quality of radiographic images. The International Commission on Radiological Pro- tection (ICRP) has both encouraged "authorized bodies" to set diagnostic reference levels (DRLs) as dose optimization tools ${ }^{4}$, consistent with regional, national or local area to which they apply and also defined the concept of effective dose $(E)^{4}$, which has become an accepted method for estimating dose and associated risks in diagnostic radiology. Some studies have been interested to dose index, like entrance surface dose (ESD) or dose area products (DAP) measurements ${ }^{5-10}$, but only few ones addressed to effective dose (E) comparison, for newborns and especially preterm infants $^{9-11}$. However, in Tunisia, the contribution of these examinations to the patient's exposure is still largely unknown.

This paper describes a comprehensive investigation on the doses delivered to newborns during their stay in the NICU of two specialized University Hospitals in Tunis. DAP, ESD as well as effective doses were evaluated, for the chest - abdomen radiologic examination. Local diagnostic reference levels (LDRLs) were derived for optimisation purposes. The 
results were compared to those published from other countries and to background radiation.

Our work has contributed to the national and international research in the field of dosimetry in neonatology, make recommendations for the optimization of radiological procedures adopted to reduce the doses received by this category of patients and opened the way for the establishment of DRLs.

\section{Methods and Materials}

This study was performed during a 4-months period in the NICU of two University Hospitals in Tunis which are specialized in neonatology and preterm infants (University Hospital A (UHA)) and in paediatrics (UHB).

The studied population included 134 neonates of both genders with different neonatal illnesses and with weights ranging from $635 \mathrm{~g}$ to $6680 \mathrm{~g}$. For this wide variation of weights $(\mathrm{W})$, neonates were categorized into six groups depending on their birth weight: $\mathrm{W}<1000 \mathrm{~g}, 1000 \mathrm{~g}<\mathrm{W}$ $<2000$ g, 2000 g $<W<3000$ g, 3000 g $<W<4000$ g, 4000 g $<$ $\mathrm{W}<5000 \mathrm{~g}$ and $\mathrm{W}>5000 \mathrm{~g}$. More than $43 \%$ patients were premature babies with extremely low birth weight $(\mathrm{W}<$ $1000 \mathrm{~g})$ and very low birth weight $(1000 \mathrm{~g}<\mathrm{W}<2000 \mathrm{~g})$, essentially admitted in the UHA. All performed radiographs were corresponding to a combined thorax-abdomen examination, not only for the treatment and follow-up but also because of the need to visualize the path of nasogastric, intravenous probes and umbilical catheters.

During their stay in the NICU, neonates and prematurely born babies underwent on average $10 \mathrm{X}$-ray examinations, with a maximum number of 75. Most of them were carried out with the baby in an incubator, the film cassette was placed under the infant. Exposure parameters $(\mathrm{kVp}$ and $\mathrm{mAs})$ were manually selected by the radiographers. For each patient and for each radiograph, data were collected such as gender, age, weight, height, thickness, tube voltage $(\mathrm{kVp})$, current time product (mAs), focus skin distance (FSD) and field size at the patient entrance surface. Radiographs were taken with two mobile X-ray units (Philips and Siemens) with an inherent filtration of $2.7 \mathrm{~mm} \mathrm{Al}$. To ensure the correct performance of the equipment, a quality control check was initially performed.
Because DRLs for neonatal radiography are expressed in terms of ESD or DAP, a DAP meter (Vacu DAP 2004), initially calibrated by the manufacturer and periodically cross calibrated in situ (using a Victoreen non-invasive X-ray test device $4000 \mathrm{M}+$ ) according to the IAEA's protocol ${ }^{12}$, was used to measure the dose in the centre of the field, by attaching it directly to the light beam diaphragm on the tube head of the mobile unit. The filtration of the DAP meter was assumed to be $0.2 \mathrm{~mm} \mathrm{Al}$.

Additionally, ESD values were deduced from DAP measurements, taking into account the backscatter factor (BSF) from the neonate and the various field sizes. A value of 1.1 was employed for the BSF for a neonate with body thickness of $5 \mathrm{~cm}$ and tube potentials in the range $50-70 \mathrm{kVp} .^{13}$

Effective doses were calculated using the PCXMC Monte Carlo program (STUK, Radiation and Nuclear Safety Authority, Helsinki, Finland) from the experimentally measured DAP. The reliability of this software for the calculation of doses to premature newborns has been proved by several authors. ${ }^{14-16}$

\section{Results and Discussion}

\section{$\mathrm{X}$-ray technique}

The exposure parameters $(\mathrm{kVp}, \mathrm{mAs}, \mathrm{FSD}$ and radiation field) are presented in Table 1 for the two NICUs. In fact, the radiographers applied a constant $\mathrm{mAs}$ value (1.6 and 3.2), with a slight variation of the tube voltage, from 55 to $60 \mathrm{kVp}$ and from 47 to $50 \mathrm{kVp}$ for the NICUs of the UHA and the $\mathrm{UHB}$, respectively.

Moreover, it is noted that the NICU of the UHB applied a low tube voltage technique with a high $\mathrm{mAs}$ setting, which will present a certain effect on increasing the patient dose. Additionally, the radiation field at the patient entrance surface has significant dimensions relative to the small size of babies. A poor collimation was undertaken by the radiographers which tends to maximize the $\mathrm{X}$-ray beam exceeding sometimes even the size of the cassette $\left(18 \times 24 \mathrm{~cm}^{2}\right)$.

Similarly, despite the difference in the population of both NICUs in terms of weight and age, these beam area dimensions do not show a high variability between the two services. Beam size affects directly the dose received by the patient and is a key element for the optimization in radiation protection.

TABLE 1: Mean and range of exposure parameters $(\mathrm{kVp}, \mathrm{mAs}$, FSD, radiation field) for the NICUs.

\begin{tabular}{ccccc}
\hline \hline & Tube voltage $(\mathrm{kVp})$ & (mAs) & FSD $(\mathrm{cm})$ & Radiation field $\left(\mathrm{cm}^{2}\right)$ \\
\hline $\begin{array}{c}\text { Mobile unit Siemens } \\
\text { (UHA) }\end{array}$ & $57[55-60]$ & 1.6 & $77[61-83]$ & $\begin{array}{c}\text { Min } 11 \times 21 \\
\text { Max 20×27 }\end{array}$ \\
$\begin{array}{c}\text { Mobile unit Philips } \\
\text { (UHB) }\end{array}$ & $48[47-50]$ & 3.2 & $64[50.5-73.5]$ & $\begin{array}{c}\text { Min 17× 21 } \\
\text { Max 26×31 }\end{array}$ \\
\hline \hline
\end{tabular}




\section{Patient doses}

The distribution of the DAP values as a function of neonatal weight is presented in Figure 1. There is a broad range with a maximum DAP value being eight to ten times the minimum, because of the differences in body sizes and to the different tube voltage and $\mathrm{mAs}$ settings between the two NICUs.

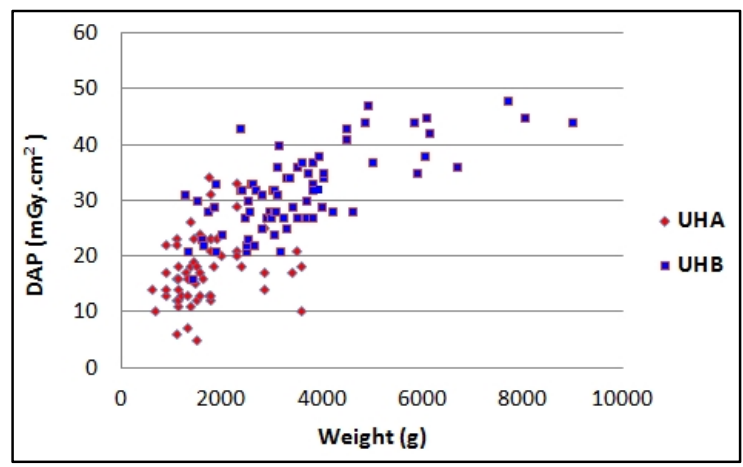

FIG. 1: Distribution of dose area products (DAP) values with neonatal weight for the two NICUs.

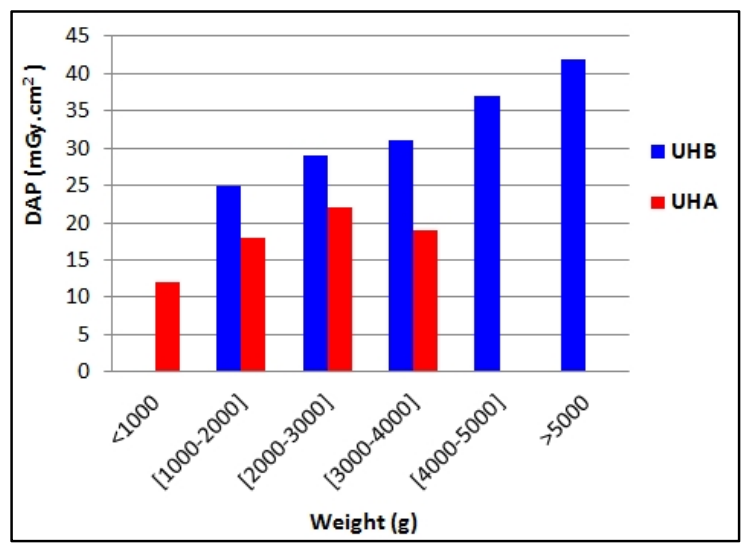

FIG. 2: Distribution of DAP (mGy.cm²) against the different neonatal weight groups in the two NICUs.

Figure 2 shows the distribution of DAP against the different neonatal weight groups in the two NICUs. We effectively observed lower DAP values in the NICU of the UHA (maternity hospital), due to the high $\mathrm{kVp}$ and low $\mathrm{mAs}$ technique used. Additionally, shorter FSD and larger field sizes caused higher DAP values in NICU of the UHB. By contrast, DAP tends to decrease slightly for the new born infants exceeding $3 \mathrm{~kg}$ in the NICU of the UHA, because the $\mathrm{kVp}$ is increased up to 60 according to patient size increase. The maximum $\mathrm{kVp}$ applied at the NICU of the UHB was too low to have an impact on DAP. However, the European Directives ${ }^{17}$ recommend a high tube voltage between 60 and 65 $\mathrm{kVp}$ for neonatal chest examination to optimize radiation doses received by the new-born. It is therefore necessary to encourage radiographers to use a standardized protocol with high voltage technique, combined to lower $\mathrm{mAs}$ values, without the loss of image quality as demonstrated by Dougeni et al. ${ }^{6}$ who prove that an increase in the tube voltage from $50 \mathrm{kV}$ to $60 \mathrm{kV}$ allows a decrease of mAs from 1.6 to 0.6 giving a maximum result of the image quality. This change of the exposure settings reduces the ESD values of $53.2 \%$ approximately.

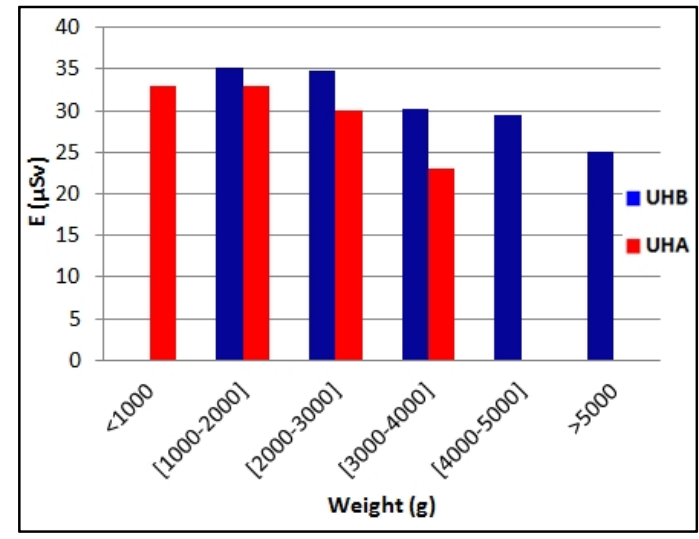

FIG. 3: Distribution of effective dose ( $\mu \mathrm{Sv})$ for the neonatal weight groups in the two NICUs.

Figure 3 presents the effective dose for different weight babies in the two NICUs. No significant difference in effective doses between the different weight categories in both NICU especially for weights ranging from $1000 \mathrm{~g}$ to $3000 \mathrm{~g}$. The poor collimation was probably the main reason for the higher effective doses, essentially for the categories of extremely low weight $(<1000 \mathrm{~g})$ and very low weight $(1000 \mathrm{~g}<\mathrm{W}<$ $2000 \mathrm{~g})$. Their organs are closer and concentrated in central $\mathrm{X}$-ray beam. By growing up, these organs spread over the whole of the beam.

The principle of optimization is essential for this category of patients. In fact, a poor collimation affects not only the image quality by increasing the parasitic radiation, but also exposes unnecessarily other organs or tissues to the primary beam. This practice is unacceptable especially in neonatology, due to the increasing sensitivity to the harmful effects of ionizing radiation.

In the same way, Figure 4 shows an example of an examination carried with a lack of collimation and then modified during the digital image processing. Some organs are unnecessarily irradiated, as the gonads and thyroid. Furthermore, the examination in this case was actually justified as chest instead of chest-abdomen.

We present in the Table 2 the mean values of the derived DAP, ESD and Effective doses per radiograph, for each weight group, in the two NICUs, in the objective of establishing Local Diagnostic Reference Levels (LDRLs) for the chest-abdomen radiologic examination. This can be an effective way for paying more attention to patient dose and optimization. We expressed LDRL for each weight category and for a patient weight sample of less than $4000 \mathrm{~g}$, presenting a sample mean weight of $1660 \mathrm{~g}$ and $2838 \mathrm{~g}$, for the NICUs of the UHA and UHB respectively. 


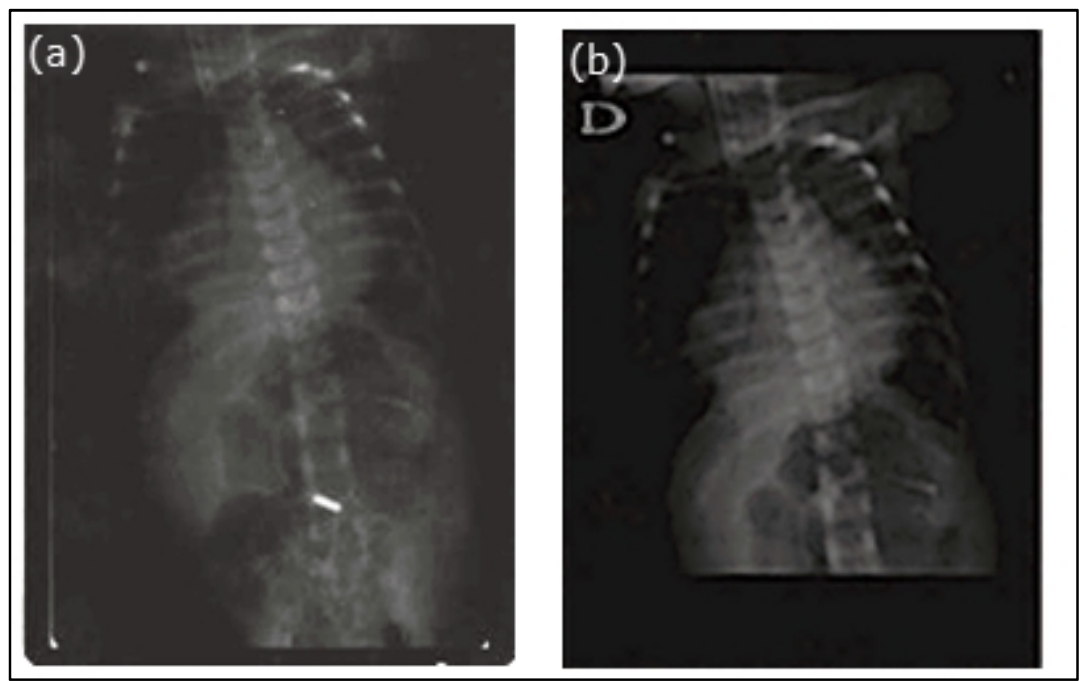

FIG. 4: Chest examinations; (a) before and (b) after digital processing.

TABLE 2: Local diagnostic reference levels expressed as mean DAP, ESD, effective dose per radiograph and standard deviation (SD) in each neonates group for the two NICUs.

\begin{tabular}{c|ccc:ccc}
\hline \hline & \multicolumn{3}{|c}{ UHA } & \multicolumn{3}{c}{ UHB } \\
\hline $\begin{array}{c}\text { Weight } \\
\text { Group }(\mathrm{g})\end{array}$ & $\begin{array}{c}\text { DAP } \\
\left(\mathrm{mGy} . \mathrm{cm}^{2}\right)\end{array}$ & $\begin{array}{c}\mathrm{ESD} \\
(\mu \mathrm{G} y)\end{array}$ & $\begin{array}{c}\mathrm{E} \\
(\mu \mathrm{Sv})\end{array}$ & $\begin{array}{c}\text { DAP } \\
\left(\mathrm{mGy} . \mathrm{cm}^{2}\right)\end{array}$ & $\begin{array}{c}\text { ESD } \\
(\mu \mathrm{Gy})\end{array}$ & $\begin{array}{c}\mathrm{E} \\
(\mu \mathrm{Sv})\end{array}$ \\
1000 & 12 & 45 & 32.7 & - & - & - \\
$1000<\mathrm{P} \leq 2000$ & 18 & 46 & 33.1 & 25 & 59 & 35.2 \\
$2000<\mathrm{P} \leq 3000$ & 22 & 50 & 30.2 & 29 & 60 & 34.8 \\
$3000<\mathrm{P} \leq 4000$ & 19 & 40 & 22.7 & 31 & 66 & 30.2 \\
$4000<\mathrm{P} \leq 5000$ & - & - & - & 37 & 69 & 29.5 \\
$>5000$ & - & - & - & 42 & 66 & 25.1 \\
Total $<4000$ & $17.6 \pm 6$ & $46.1 \pm 14$ & $30.6 \pm 10$ & $29.1 \pm 7$ & $64.1 \pm 13$ & $32.6 \pm 7$ \\
\hline \hline
\end{tabular}

TABLE 3: Comparison of mean DAP, ESD and E with previous studies.

\begin{tabular}{|c|c|c|c|c|}
\hline Reference & Weight (g) & DAP (mGy.cm²) & $\operatorname{ESD}(\mu \mathrm{Gy})$ & $\mathrm{E}(\mu \mathrm{Sv})$ \\
\hline This study & $2254^{*}[600-4000]$ & 23.4 & 55.2 & 31.6 \\
\hline J. Dabin et al., $2013^{5}$ & [600-4000] & 11 & 43 & - \\
\hline Dougeni et al., $2007^{6}$ & {$[600-4000]$} & 8.1 & 34 & - \\
\hline Smans et al. , $2008^{7}$ & $>2500$ & - & 52 & - \\
\hline Smans et al., $2008^{7}$ & [1000-2500] & - & 28.33 & - \\
\hline Hart et al., $2000^{8}$ & $\mathrm{NA}^{* *}$ & - & 50 & - \\
\hline Jones et al., $2001^{9}$ & Phantom of $2500 \mathrm{~g}$ & 18.7 & 71.5 & 35.5 \\
\hline Armpilia et al., $2002^{10}$ & 1100 & 5.5 & 35 & 9.2 \\
\hline CEC, $1996^{17}$ & - & - & 80 & - \\
\hline
\end{tabular}

*: Mean value; NA**: Not available $^{*}$

Table 3 provides patients data; mean DAP, ESD and Effective doses comparison with former similar investigations. ${ }^{5-10}$ This comparison offers a wide variation of dose index (Mean DAP, Mean ESD or DRL) resulting from three radiological examinations (chest, abdomen and chest-abdomen) and for different weight categories.

In terms of DAP, our values are higher than those reported by the previous studies and especially the results of Dabin et al. ${ }^{5}$ and Dougeni et $a l .{ }^{6}$, for which we consider that we have the same weight sample (<4000 g). This confirms the need of the optimization of the irradiation for this category of pa- tient. Even if the LDRLs are in agreement with CEC ${ }^{17}$, $\mathrm{NRPB}^{8}$ for neonatal radiography, the difference between the two NICUs encourages the stakeholder to contribute to an agreed protocol among the departments using mobile X-ray units in the NICUs.

Few studies have focused on the effective dose. The results of our study, in term of E, are similar to the value provided by Jones et al..$^{9}$ who used a phantom of $2500 \mathrm{~g}$ weight while the value obtained in our study is almost identical for all weight categories. This is due to the same exposure and irradiation parameters used for a newborn of $600 \mathrm{~g}$ and that of $3000 \mathrm{~g}$. 


\section{Estimation of risk}

The major concern regarding the use of ionizing radiation on the newborn is the risk of inducing a fatal childhood cancer. ${ }^{4}$ Since the majority of neonates in intensive care are preterm, fatal risk factors were used to estimate the risk of exposure to ionizing radiation. ${ }^{9}$

However, there is some uncertainty about the most appropriate fatal risk factor used. According to the $\mathrm{ICRP}^{4}$, the overall risk of inducing a fatal cancer in the first decade of life, following pre-natal exposure, has been estimated to be between $2.8 \times 10^{-2} \mathrm{~Sv}^{-1}$ and $13 \times 10^{-2} \mathrm{~Sv}^{-1}$. Therefore, using these factors multiplied by the mean ED, the risk of childhood cancer from a single radiograph would vary from 0.9 to $4.1\left(^{\prime} 10^{-6}\right)$. These estimates are similar to the values published by Jones et al. ${ }^{9}$ of 4.6 using the higher factor to calculate the worst case scenario.

To demonstrate the probability of damaging effects of radiography is to compare the cumulative effective dose from the total radiographs with a time equivalent amount of yearly natural background radiation. Thus, the mean cumulative $\mathrm{E}$ from 10 undergone radiographs on a preterm during his stay in the NICUs is estimated to $316 \mu \mathrm{Sv}$ equivalent to about 49 days of natural exposure if we consider a natural background of $2.4 \mathrm{mSv} /$ year in the world. ${ }^{1}$ Although this value is well below the yearly natural background and the examinations are absolutely justified and in accordance with patient benefit, neonatal radiological examinations deserve special consideration in view of the increased radiation risk.

\section{Conclusion}

In order to prevent the increasing risk of long term effects to neonates, a specific population which undergoes multiple diagnostic examinations during a short period, several actions should be taken at a national level as well as international levels. DRLs for neonates in the NICUs of the region of Tunis were in good compliance with the CEC and the NRPB values but slightly higher than those reported in more recent studies.

However, the findings show that the use of high tube voltage techniques and an adapted collimation could lead to further reductions in the neonatal doses, especially to premature babies. It is necessary that the system be optimized for performing radiographic examinations. In fact, the establishment of LDRL in each department can be an effective way for paying attention to patient dose and optimization and reinforce the role of the medical physicists in the field of patient protection and safety during medical exposure.

\section{Conflict of interest}

The authors declare that they have no conflicts of interest. The authors alone are responsible for the content and writing of the paper.

\section{References}

1. United Nations Scientific Committee on the effects of atomic radiation (UNSCEAR): Sources, effects and risks of ionizing radiation, UNSCEAR 2000 report, volume II: effects NY: United Nations, 2000.

2. Almen AJ, Mattsson S, On the calculation of effective dose to children and adolescents. J Radiol Prot.1996; 16: 81-9.

3. International Commission on Radiological Protection. Radiological Protection and Safety in Medicine, ICRP, Publication 73. Ann. ICRP 26(1-47) (Oxford: Elsevier Science Ltd) 1996.

4. International Commission on Radiation Protection 1990. Recommendations of the Internationa Commission on Radiation Protection. ICRP publication 60. Oxford: Pergamon, 1991.

5. Dabin J, Struelens L, Vanhavere F. Evaluation of the doses delivered to premature babies in the Belgian Neonatal Intensive Care Units. Radiat Prot Dosimetry. 2013;158:28-35.

6. Dougeni ED, Delis HB, Karatza AA, et al. Dose and image quality optimization in neonatal radiography. Br J Radiol 2007;80: 807-15.

7. Smans K, Struelens L, Smet M, et al. Patient dose in neonatal units. Radiat Prot Dosimetry. 2008; 131:143-7.

8. Hart D, Wall BF, Shrimpton PC, et al. Reference doses and patient size in paediatric radiology, NRPB-R318. Chitton, UK: NRPB, 2000.

9. Jones NF, Palarm TW, Negus IS, Neonatal chest and abdominal radiation dosimetry: a comparison of two radiographic techniques. Br J Radiol. 2001; 74: 920-5.

10. Armpilia, CI, Fife IAJ, Croasdale PL, Radiation dose quantities and risk in neonates in a special baby care unit. Br J Radiol. 2002;75:590-5.

11. Puch-Kapst K, Juran R, Stoever B, Wauer RR. Radiation exposure in 212 very low and extremely low birth weight infant. Pediatrics. 2009; 124: 1556-64.

12. International Atomic Energy Agency (IAEA). Dosimetry in Diagnostic Radiology: An International Code of Practice. TRS N 457, Vienna 2007. 
13. Hart D, Jones DG, Wall BF. Coefficients for estimating effective doses from paediatric X-ray examinations. Nrpb Reports: Reports R279. Chilton: HMSO, 1996.

14. Servomaa A, Tapiovaara M. Organ dose calculation in medical X-ray examinations by the program PCXMC. Radiat Prot Dosim. 1998;80:213-9.

15. Ma H, Elbakri IA, Reed M. Estimation of organ and effective doses from newborn radiography of the chest and abdomen. Radiat Prot Dosimetry. 2013; 156:160-7.

16. Smans K, Tapiovaara M, Cannie M, et al. Calculation of organ doses in $\mathrm{x}$-ray examinations of premature babies. Med Phys. 2008;35:556-68.

17. Commission of European Communities. European guidelines on quality criteria for diagnostic radiographic images. Report EUR 16261. Luxemburg: Office for Official Publications of the European Communities, 1996. 